Check for updates

Cite this: RSC Adv., 2019, 9, 10781

Received 3rd December 2018

Accepted 25th March 2019

DOI: $10.1039 / \mathrm{c} 8 \mathrm{ra09926j}$

rsc.li/rsc-advances

\section{The interaction of dietary flavonoids with xanthine oxidase in vitro: molecular property-binding affinity relationship aspects}

\author{
Mengmeng Yuan, $\dagger^{\mathrm{ac}}$ Yi Liu, $\dagger^{\mathrm{a}}$ Aiping Xiao, ${ }^{a}$ Juan Leng, ${ }^{a}$ Liping Liao, ${ }^{a}$ Lei Ma*b \\ and Liangliang Liu (D) *a
}

\begin{abstract}
The molecular property-affinity relationships of dietary flavonoids binding to xanthine oxidase were investigated in vitro by comparing the binding constants obtained from a fluorescence-quenching method. The inhibitions of dietary flavonoids on xanthine oxidase were also investigated and analyzed, revealing that the binding process was influenced by the structural differences of the flavonoids under investigation. For example, methylation and hydroxylation at the 7- and 5-positions weakened the binding affinities, while hydroxylation at the 3 - and $3^{\prime}$-positions mostly improved binding affinities. Glycosylation and hydrogenation of the $\mathrm{C}_{2}=\mathrm{C}_{3}$ double bond also increased affinities for xanthine oxidase. In addition, galloylated catechins showed higher binding affinities than non-galloylated catechins. Trends in the binding affinities and inhibition of flavonoids during structure modifications were summarized. Affinities for xanthine oxidase and inhibition on xanthine oxidase changed in the opposite direction during the methylation and hydroxylation of flavonoids in the A ring, and the glycosylation and hydrogenation of $\mathrm{C}_{2}=\mathrm{C}_{3}$. However, affinities and inhibition for xanthine oxidase changed in the same direction during the methylation and hydroxylation of flavonoids in the $\mathrm{B}$ ring.
\end{abstract}

\section{Introduction}

Dietary flavonoids exist widely in many plant foods, such as fruits, vegetables, nuts, and tea., ${ }^{\mathbf{1} 2}$ Over 10000 flavonoids have been separated and identified from plants, including anthocyanidins, flavones, flavonols, flavanones, and isoflavones. ${ }^{3}$ Investigation of flavonoids from dietary sources has attracted considerable interest because of their nutritional and medical effects in humans. Most of their bioactivity is related to their structure; ${ }^{4,5}$ for example, the presence of a 2,3 double bond in conjugation with a 4-oxo group and a catechol unit (1,2-dihydroxybenzene) is usually required for antioxidant activity in flavonoids. Methylation, hydroxylation, and glycosylation of flavonoids also affect their absorption, metabolism, and bioactivities in vivo. ${ }^{6,7}$ Furthermore, structural differences in flavonoids strongly affect the binding process with plasma proteins, which are linked to the chemistry of the $\mathrm{C}$ ring, the number and distribution of hydroxyl groups, and their substitutions on the $\mathrm{A}$ and $\mathrm{B}$ rings. ${ }^{8}$ According to reports, the

\footnotetext{
${ }^{a}$ Institute of Bast Fiber Crops, Chinese Academy of Agricultural Sciences, Changsha 410205, China. E-mail: liuliangliang@caas.cn

${ }^{b}$ Zhengzhou Research Base, State Key Laboratory of Cotton Biology, Zhengzhou University, China. E-mail: malei@caas.cn

${ }^{c}$ College of Chemistry and Chemical Engineering, Central South University, Changsha 410083, China

$\dagger$ These authors contributed equally to this work.
}

methylation of the free hydroxyl groups in the flavonoids dramatically increases their intestinal absorption and metabolic resistance and transport of flavonoids. ${ }^{9}$

Fluorescence-quenching spectroscopy is an important method to determine the binding parameters between ligands and bio-macromolecules. ${ }^{\mathbf{1 0 1 1}}$ It refers to the decrease in the quantum yield of fluorescence from a fluorophore induced by a variety of molecular interactions with a quencher molecule under excitation at $280 \mathrm{~nm} .{ }^{12}$ In recent years, studies on fluorescence quenching have been widely reported in the literature. Frazier et al. investigated the interaction of four different flavonoids with bovine serum albumin (BSA) by quenching the intrinsic tryptophan fluorescence in order to study the chemical associations. ${ }^{13}$ Xiao et al. investigated the interaction of puerarin and BSA by means of fluorescence spectroscopy. ${ }^{\mathbf{1 4}}$ Shao et al. investigated the structure-affinity relationship in the interactions of different oleanane-type triterpenoids with BSA by spectroscopic analysis. In addition to the research focusing on the binding process, the structure-affinity relationships of bioactive compounds for proteins such as common rat plasma proteins, common human plasma proteins, $\gamma$-globulin, total plasma proteins, BSA and HSA were also investigated..$^{15-19}$

Xanthine oxidase could catalyze the oxidation of hypoxanthine to xanthine and further catalyze the oxidation of xanthine to uric acid, which plays a crucial role in gout. ${ }^{20}$ Excess accumulation of uric acid in serum will lead to hyperuricemia, which is a risk factor for cardiovascular disease, gout and other 
metabolic disorders. ${ }^{21}$ Thus, the investigation of xanthine oxidase inhibitors could be an effective strategy in the prevention of these metabolic disorders. ${ }^{22}$ Fluorescence-quenching spectroscopy was also used in research of xanthine oxidase. By analyzing fluorescence parameters, considerable information relating to structural changes in enzymes could be obtained. ${ }^{23}$ Rashidi et al. first studied the interaction of quercetin and bovine milk xanthine oxidase using a fluorescencequenching method..$^{24}$ Lin et al. reported that five dietary flavonoids could interact with xanthine oxidase at more than one binding site through fluorescence-quenching data. ${ }^{20}$ However, there were no reports on structure-affinity relationships in the interactions of different flavonoids with xanthine oxidase.

In our previous research, xanthine oxidase inhibitors from natural plants were screened and identified. ${ }^{25,26}$ The binding properties of tectoridin, daidzin, ononin, biochanin A, gardenin $\mathrm{B}$, and eupatorin for xanthine oxidase were also studied. Based on these results, we became interested in detailed research on the binding between activity compounds and xanthine oxidase, and a series of flavonoids with similar structures were selected for research. In the present study, xanthine oxidase was used as a modeling protein in order to investigate the interaction between small molecules and protein, and 35 dietary flavonoids (shown in Table 1) were selected to investigate the interaction with xanthine oxidase.

\section{Experimental}

\subsection{Materials}

Xanthine oxidase powder was purchased from Yuanye Biotechnology Co. (Shanghai, China). Flavone (99.0\%), 7-OH flavone $(98.0 \%)$, chrysin $(98.0 \%)$, biochanin A $(98.0 \%)$, naringin (98.0\%), and myricetin (98.0\%) were bought from Alfa Aesar (Thermo Fisher Scientific, MA, USA). Formononetin (99.0\%) and genistein (99.0\%) were acquired from Acros Organics (Fisher Scientific, PA, USA). Baicalin (98.0\%), baicalein (98.0\%), wogonin (98.0\%), apigenin (98.0\%), luteolin (98.0\%), hispidulin (98.0\%), tangeretin (98.0\%), nobiletin (98.0\%), galangin (98.0\%), kaempferide (98.0\%), kaempferol (98.0\%), kaempferitrin $(98.0 \%)$, quercetin $(98.0 \%)$, fisetin $(98.0 \%)$, rutin $(98.0 \%)$,

Table 1 Chemical structures of the 35 investigated dietary flavonoids

\begin{tabular}{|c|c|c|c|c|c|}
\hline \multirow[b]{2}{*}{ Subclass } & \multirow[b]{2}{*}{ Name } & \multicolumn{3}{|l|}{ Substitutions } & \multirow[b]{2}{*}{ Structure } \\
\hline & & $\mathrm{OH}$ & $\mathrm{OCH}_{3}$ & Others & \\
\hline \multirow{11}{*}{ Flavones } & Flavone & & & & \\
\hline & 7-OH flavone & 7 & & & \\
\hline & Chrysin & 5,7 & & & \\
\hline & Baicalin & 5,6 & & & 8 \\
\hline & Baicalein & $5,6,7$ & & & \\
\hline & Wogonin & 5,7 & 8 & & \\
\hline & Apigenin & $5,7,4^{\prime}$ & & & 5 \\
\hline & Luteolin & $5,7,3^{\prime}, 4^{\prime}$ & & & \\
\hline & Hispidulin & $5,7,4^{\prime}$ & 6 & & \\
\hline & Tangeretin & & $5,6,7,8,4^{\prime}$ & & \\
\hline & Nobiletin & & $5,6,7,8,4^{\prime}, 5^{\prime}$ & & \\
\hline \multirow{8}{*}{ Flavonols } & Galangin & $3,5,7$ & & & \\
\hline & Kaempferide & $3,5,7$ & $4^{\prime}$ & & \\
\hline & Kaempferol & $3,5,7,4^{\prime}$ & & & \\
\hline & Kaempferitrin & $5,4^{\prime}$ & & 3,7-Dirhamnoside & \\
\hline & Quercetin & $3,5,7,3^{\prime}, 4^{\prime}$ & & & \\
\hline & Myricetin & $3,5,7,3^{\prime}, 4^{\prime}, 5^{\prime}$ & & & \\
\hline & Fisetin & $3,7,3^{\prime}, 4^{\prime}$ & & & \\
\hline & Rutin & $5,7,3^{\prime}, 4^{\prime}$ & & 3- $a$-L-Rham-1,6-D-Glc & \\
\hline \multirow{6}{*}{ Isoflavones } & Formononetin & 7 & $4^{\prime}$ & & \\
\hline & Genistein & $5,7,4^{\prime}$ & & & \\
\hline & Daidzein & $7,4^{\prime}$ & & & \\
\hline & Biochanin A & 5,7 & $4^{\prime}$ & & \\
\hline & Tectorigenin & $5,7,4^{\prime}$ & 6 & & \\
\hline & Puerarin & $7,4^{\prime}$ & & 8-C-Glucoside & \\
\hline \multirow[b]{3}{*}{ Flavanone } & Dihydromyricetin & $3,5,7,3^{\prime}, 4^{\prime}, 5^{\prime}$ & & & \\
\hline & Naringenin & $5,7,4^{\prime}$ & & & \\
\hline & Naringin & $5,4^{\prime}$ & & 7-Neohesperidose & \\
\hline \multirow{7}{*}{ Flavanonol } & $\mathrm{C}(2,3-$ trans $)$ & $3,5,7,4^{\prime}, 5^{\prime}$ & & & \\
\hline & $\mathrm{EC}(2,3-c i s)$ & $3,5,7,4^{\prime}, 5^{\prime}$ & & & \\
\hline & ECG $(2,3-c i s)$ & $5,7,4^{\prime}, 5^{\prime}$ & & 3-Gallate & \\
\hline & EGC $(2,3-c i s)$ & $3,5,7,3^{\prime}, 4^{\prime}, 5^{\prime}$ & & & \\
\hline & EGCG $(2,3-c i s)$ & $5,7,3^{\prime}, 4^{\prime}, 5^{\prime}$ & & 3-Gallate & \\
\hline & GC (2,3-trans) & $3,5,7,3^{\prime}, 4^{\prime}, 5^{\prime}$ & & & \\
\hline & GCG (2,3-trans) & $5,7,3^{\prime}, 4^{\prime}, 5^{\prime}$ & & 3-Gallate & \\
\hline
\end{tabular}


daidzein (98.0\%), tectorigenin $(98.0 \%)$, puerarin $(98.0 \%)$, dihydromyricetin (98.0\%), naringenin (98.0\%), catechin (C; 98.0\%), (-)-gallocatechin (GC; 98.0\%), epicatechin, (EC; 98.0\%), (-)-epicatechin gallate (ECG; 98.0\%), (-)-epigallocatechin (EGC; 98.0\%), (-)-epigallocatechin gallate (EGCG; 98.0\%), and (-)-gallocatechin gallate (GCG; 98.0\%) were commercially purchased from Yuanye Biotechnology Co. (Shanghai, China). High-performance liquid chromatography (HPLC) grade acetonitrile was purchased from Merck KGaA (Darmstadt, Germany). Ultrapure water (18.2 $\mathrm{M} \Omega \mathrm{cm}$ resistivity) was obtained from an ELGA water purification system (ELGA Berkefeld, Veolia, Germany). All other reagents and solvents were of analytical grade.

\subsection{Apparatus}

Fluorescence spectra were recorded on a fluorometer (Hitachi F7000, Tokyo, Japan) using $1.0 \mathrm{~cm}$ of quartz cuvette. The thermostatic control of incubation was completed with a thermostat water bath (MS7-H550-Pro, DLAB Scientific Inc., Beijing, China) controlled at $25{ }^{\circ} \mathrm{C}$. The air conditioner was set at $25{ }^{\circ} \mathrm{C}$ to minimize interference during detection. Ultraviolet-visible (UVvis) spectra and inhibition tests were recorded on an UV-vis spectrophotometer using $1.0 \mathrm{~cm}$ of quartz cuvette (UV2700, Shimadzu, Kyoto, Japan). Qualitative analysis of dietary flavonoids was completed by HPLC analysis on an Agilent 1260 HPLC system (Agilent Technologies, Santa Clara, CA, USA) equipped with a $\mathrm{C}_{18}$ reverse-phase column (Waters Xbridge ${ }^{\mathrm{TM}}$, $250 \mathrm{~mm} \times 4.6 \mathrm{~mm}$ i.d., $5 \mu \mathrm{m}$, Milford, MA, USA).

\subsection{Inhibition test}

First, $20 \mu \mathrm{L}$ of xanthine oxidase solution $\left(3.70 \mathrm{nmol} \mathrm{L}^{-1}\right)$ and a sample with a different volume (10 to $100 \mu \mathrm{L})$ were mixed in a quartz cuvette, ${ }^{26}$ and water was added to make the total volume of mixture up to $1.0 \mathrm{~mL}$. Then, $1.0 \mathrm{~mL}$ of xanthine solution $\left(0.45 \mathrm{mmol} \mathrm{L}^{-1}\right)$ was added to start the enzymatic reaction. The cuvette was rapidly moved into a UV-vis spectrophotometer, and the absorbance at $295 \mathrm{~nm}$ was monitored. The same amount of water was used as in the blank, under the same conditions. The inhibition of xanthine oxidase could be calculated using the following formula:

$$
\text { Inhibition } \%=\left(1-\Delta A_{\mathrm{s}} / \Delta A_{\mathrm{b}}\right) \times 100 \%
$$

where $\Delta A_{\mathrm{s}}$ and $\Delta A_{\mathrm{b}}$ are the increase of absorbance for the sample and blank, respectively. Inhibition of the sample was expressed as the concentration of sample needed to inhibit 50\% of enzymatic activity $\left(\mathrm{IC}_{50}\right)$. The detection limit of xanthine oxidase activity was $1.0 \mathrm{U} \mathrm{L}^{-1}$. The detection limit of related inhibitors was in the range $0.05 \mathrm{nmol} \mathrm{L}^{-1}$ to $5.0 \mathrm{mmol} \mathrm{L}^{-1}$. All assays were performed with three replicates.

\subsection{Fluorescence spectra analysis}

The fluorescence spectra of xanthine oxidase and the quenching effects of samples were performed according to procedures reported in previous literature. ${ }^{27,28}$ Various volumes of samples (0$100 \mu \mathrm{L}, 1.0 \mathrm{mmol} \mathrm{L}^{-1}$ in methanol) were mixed with $2.0 \mathrm{~mL}$ of xanthine oxidase solution $\left(1.0 \mu \mathrm{mol} \mathrm{L}{ }^{-1}\right.$ in phosphate buffered saline). After incubation at $25{ }^{\circ} \mathrm{C}$ for $5 \mathrm{~min}$, the fluorescence emission spectra of samples were recorded from 300 to $450 \mathrm{~nm}$ under an excitation wavelength at $280 \mathrm{~nm}$ using a fluorometer. All assays were performed with three replicates.

\subsection{Data processing}

The fluorescence quenching of xanthine oxidase with samples were described by the Stern-Volmer formula shown below: ${ }^{\mathbf{1 8 2 9}}$

$$
F_{0} / F=1+K_{\mathrm{q}} \tau_{0}[\mathrm{Q}]=1+K_{\mathrm{sv}}[\mathrm{Q}]
$$

where $F_{0}$ and $F$ are the fluorescence intensity of xanthine oxidase at $335 \mathrm{~nm}$ in the absence and presence of sample, [Q] is the concentration of the sample, $K_{\mathrm{q}}$ is the quenching rate constant, $\tau_{0}$ is the average lifetime $(6.2 \mathrm{~ns})$, and $K_{\mathrm{sv}}$ is the dynamic quenching constant. The binding constants were calculated according to the double-logarithm formula:

$$
\log _{10}\left[\left(F_{0}-F\right) / F\right]=\log _{10} K_{\mathrm{a}}+n \log _{10}[\mathrm{Q}]
$$

where $K_{\mathrm{a}}$ is the binding constant and $n$ is the number of binding sites per enzyme molecule. According to formulas (2) and (3), the values of " $F_{0} / F$ " and " $\left(F_{0}-F\right) / F$ " could be observed in each "[Q]" of samples. Then, the linear regression equation between the " $F_{0} / F$ " values and "[Q]" values was obtained, and the slope means " $K_{\mathrm{sv}}$ ". The linear regression equation between the " $\log _{10}\left[\left(F_{0}-F\right) / F\right]$ " and " $\log _{10}[\mathrm{Q}]$ " values could be obtained. The slope means " $n$ " and the means " $\log _{10} K_{\mathrm{a}}$ ".

\subsection{Statistical analysis}

The experiments under each condition were performed in triplicate. The experimental errors were less than $5.0 \%$. The results were presented as the means \pm standard deviation (SD), and analyzed by one-way analysis of variance (ANOVA) and Duncan's post hoc analysis. $p<0.05$ was considered to be statistically significant.

\section{Results and discussion}

\subsection{Inhibition of flavonoids on xanthine oxidase}

The inhibitions of 35 dietary flavonoids on xanthine oxidase were investigated. Inhibition was expressed as $\mathrm{IC}_{50}$ values in Table 2, and all 35 dietary flavonoids exhibited some degree of inhibition on xanthine oxidase except kaempferide. Quercetin showed the highest inhibitory activity at $0.0001 \mu \mathrm{mol} \mathrm{L}^{-1}$, while puerarin showed the lowest inhibitory activity at $2.1074 \mu \mathrm{mol}$ $\mathrm{L}^{-1}$. Therefore, these dietary flavonoids showed good inhibition on xanthine oxidase.

\subsection{Fluorescence-quenching effects}

Fluorescence quenching is a common bioanalytical method widely used in investigating the interaction between biological macromolecules and small molecules. The fluorescence emission spectra of xanthine oxidase with the addition of various dietary flavonoids were monitored, and six of these flavonoids 
Table 2 Affinities, inhibitions and property parameters of flavonoids for xanthine oxidase ${ }^{a}$

\begin{tabular}{|c|c|c|c|c|c|c|c|}
\hline \multirow[b]{2}{*}{ Name } & \multirow[b]{2}{*}{ Inhibition $\mathrm{IC}_{50}\left(\mu \mathrm{mol} \mathrm{L}{ }^{-1}\right)$} & \multicolumn{4}{|l|}{ Affinity } & \multirow[b]{2}{*}{ TPSA $\left(\AA^{2}\right)$} & \multirow[b]{2}{*}{$X \log P_{3}$} \\
\hline & & $K_{\text {sv }}\left(10^{6}\right)$ & $K_{\mathrm{q}}\left(10^{13}\right)$ & $\log _{10} K_{\mathrm{a}}$ & $n$ & & \\
\hline Flavone & 0.3464 & 0.0552 & 0.8903 & 6.3124 & 1.3695 & 26.3 & 3.6 \\
\hline 7-OH flavone & 0.1505 & 0.0360 & 0.5806 & 4.4265 & 0.9676 & 46.5 & 3.6 \\
\hline Chrysin & 0.0138 & 0.0195 & 0.3145 & 2.5792 & 0.6025 & 66.8 & 2.1 \\
\hline Baicalin & 0.0267 & 0.3668 & 5.9161 & 8.9699 & 1.8782 & 183.0 & 1.1 \\
\hline Baicalein & 0.0258 & 0.1981 & 3.1951 & 7.3561 & 1.5312 & 87.0 & 1.7 \\
\hline Wogonin & 0.0174 & - & - & - & - & 76.0 & 3.0 \\
\hline Apigenin & 0.0007 & 0.0285 & 0.4596 & 2.1428 & 0.4524 & 87.0 & 1.7 \\
\hline Luteolin & 0.0003 & 0.0926 & 1.4935 & 6.2828 & 1.3087 & 107.0 & 1.4 \\
\hline Hispidulin & 0.0159 & 0.0989 & 1.5951 & 6.1617 & 1.2571 & 96.2 & 1.7 \\
\hline Tangeretin & 1.7885 & 0.1498 & 2.4161 & 5.1591 & 1.0022 & 72.4 & 3.0 \\
\hline Nobiletin & 0.2647 & 0.9637 & 15.5435 & 14.019 & 2.8371 & 81.7 & 3.0 \\
\hline Galangin & 0.0090 & 0.0276 & 0.4451 & 4.0235 & 0.9038 & 87.0 & 2.3 \\
\hline Kaempferide & ND & - & - & - & - & 96.2 & 2.2 \\
\hline Kaempferol & 0.0050 & 0.0225 & 0.3629 & 4.5889 & 1.0535 & 107.0 & 1.9 \\
\hline Kaempferitrin & 0.2788 & 0.0929 & 1.4983 & 7.0817 & 1.5204 & 225.0 & -0.1 \\
\hline Quercetin & 0.0001 & 0.0477 & 0.7693 & 4.9449 & 1.0614 & 127.0 & 1.5 \\
\hline Myricetin & 0.0086 & 0.0294 & 0.4741 & 4.7891 & 1.0764 & 148.0 & 1.2 \\
\hline Fisetin & 0.0063 & 0.0729 & 1.1758 & 6.4458 & 1.3663 & 107.0 & 2.0 \\
\hline Rutin & 1.0333 & 0.0532 & 0.8580 & 5.9546 & 1.2904 & 266.0 & -1.3 \\
\hline Formononetin & 0.0719 & 0.0147 & 0.2370 & 2.8576 & 0.6866 & 55.8 & 2.8 \\
\hline Genistein & 0.2508 & 0.0537 & 0.8661 & 6.3981 & 1.3911 & 87.0 & 2.7 \\
\hline Daidzein & 0.1799 & 0.0230 & 0.3709 & 5.4529 & 1.2554 & 66.8 & 2.5 \\
\hline Biochanin A & 0.1570 & 0.0359 & 0.5790 & 0.3808 & 0.7052 & 76.0 & 3.0 \\
\hline Tectorigenin & 0.1426 & 0.0644 & 1.0387 & 3.0234 & 0.6283 & 96.2 & 2.6 \\
\hline Puerarin & 2.1074 & 0.0290 & 0.4677 & 5.2024 & 1.1801 & 157.0 & 0.0 \\
\hline Dihydromyricetin & 0.0428 & 0.0470 & 0.7580 & 5.6369 & 1.2262 & 148.0 & 1.1 \\
\hline Naringenin & 0.1436 & 0.0568 & 0.9161 & 5.8127 & 1.2510 & 87.0 & 2.4 \\
\hline Naringin & 0.3267 & 0.0846 & 1.3645 & 7.6780 & 1.6838 & 225.0 & -0.5 \\
\hline C (2,3-trans) & 0.2591 & - & - & - & - & 110.0 & 0.4 \\
\hline $\mathrm{EC}(2,3-c i s)$ & 0.2774 & - & - & - & - & 110.0 & 0.4 \\
\hline ECG (2,3-cis) & 0.2610 & 0.0193 & 0.3112 & 4.7240 & 1.099 & 177.0 & 1.5 \\
\hline EGC (2,3-cis) & 0.2510 & - & - & - & - & 131.0 & 0.0 \\
\hline EGCG $(2,3-c i s)$ & 0.2805 & 0.0226 & 0.3645 & 5.3153 & 1.2461 & 197.0 & 1.2 \\
\hline GC (2,3-trans $)$ & 0.2819 & - & - & - & - & 131.0 & 0.0 \\
\hline GCG $(2,3-$ trans $)$ & 0.0063 & 0.0178 & 0.2871 & 4.3766 & 1.0301 & 197.0 & 1.2 \\
\hline
\end{tabular}

are shown in Fig. 1. Xanthine oxidase solution showed an apparent fluorescence emission peak at $335 \mathrm{~nm}$, and the fluorescence intensities reduced gradually with increasing addition of dietary flavonoids in XO solution.

In order to evaluate and compare the quenching effects of 35 dietary flavonoids, Stern-Volmer plots for fluorescence quenching were used to find further quenching information. The Stern-Volmer plots of six flavonoids are shown in Fig. 2, and the calculated parameters of 35 dietary flavonoids are demonstrated in Table 2. As shown in Fig. 2 and Table 2, the degrees of fitting of the Stern-Volmer plots were greater than 0.9 , reflecting that the calculations of $K_{\mathrm{sv}}$ and $K_{\mathrm{q}}$ were acceptable. High linearities of the Stern-Volmer plots indicated that one quenching mode played a key role in the interaction, and $K_{\mathrm{q}}$ was the factor indicating the efficiency of quenching. When $K_{\mathrm{q}}$ is apparently greater than $2.0 \times 10^{10} \mathrm{~L}$ $\mathrm{mol}^{-1} \mathrm{~s}^{-1}$, it could be considered as static quenching; ${ }^{30}$ otherwise it would be considered dynamic quenching. In this study, the values of $K_{\mathrm{q}}$ of 35 dietary flavonoids were much greater than $2.0 \times 10^{10} \mathrm{~L} \mathrm{~mol}^{-1} \mathrm{~s}^{-1}$. The lowest value of $K_{\mathrm{q}}$ was $0.237 \mathrm{~L} \mathrm{~mol}^{-1} \mathrm{~s}^{-1}$ for formononetin. Therefore, based on this, the quenching of 35 dietary flavonoids could be considered as static quenching.

\subsection{Binding parameters}

For static quenching, the number of binding sites per protein molecule $(n)$ and binding constants $\left(K_{\mathrm{a}}\right)$ could be calculated by fitting the double-logarithm curves. The values of $n$ and $K_{\mathrm{a}}$ are also shown in Table 2 and the plots of $\log \left[\left(F_{0}-F\right) / F\right]$ versus $\log [\mathrm{Q}]$ of six representative flavonoids are shown in Fig. 3. The degrees of fitting of these calculations were higher than 0.9882 , and the linearity of curves indicated that the calculations were acceptable. ${ }^{31}$ The plots of $\log _{10} K_{\mathrm{a}}$ versus $n$ of flavonoids are shown in Fig. 4 . The values of $\log _{10} K_{\mathrm{a}}$ were linear to the number of binding sites $(n)$ (fitting curve: $\left.\log _{10} K_{\mathrm{a}}=0.182 n+0.212, R^{2}=0.9498\right)$. This indicates that the equation used in this study was suitable for the interaction between flavonoids and xanthine oxidase. Thirty-five dietary 

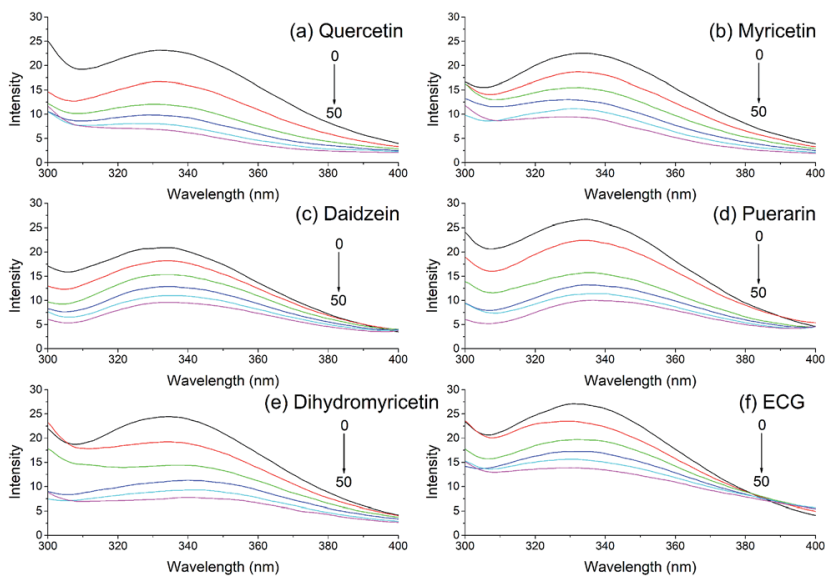

Fig. 1 Quenching effects of (a) quercetin, (b) myricetin, (c) daidzein, (d) puerarin, (e) dihydromyricetin, and (f) ECG (2,3-cis) on xanthine oxidase fluorescence spectra. $\lambda_{\mathrm{ex}}=280 \mathrm{~nm}$; xanthine oxidase, 1.0 $\mu \mathrm{mol} \mathrm{L}{ }^{-1}$; the addition of sample was 0 (black), 20 (red), 40 (green), 60 (blue), 80 (light blue), and $100 \mu \mathrm{L}$ (purple).

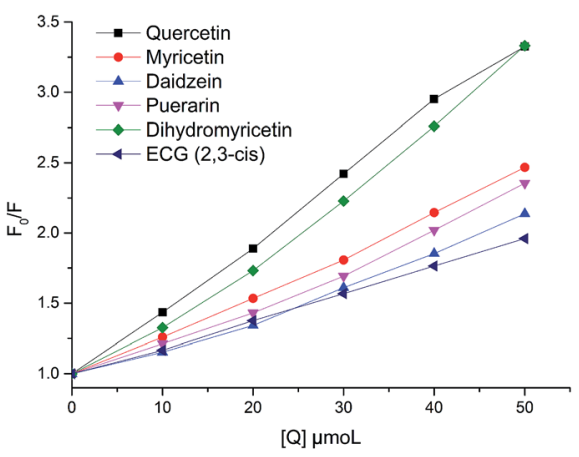

Fig. 2 Stern-Volmer plots for xanthine oxidase fluorescence quenching by quercetin, myricetin, daidzein, puerarin, dihydromyricetin, and ECG (2,3-cis). The degrees of fitting were 0.9978 for quercetin, 0.9972 for myricetin, 0.9893 for daidzein, 0.9895 for puerarin, 0.9906 for dihydromyricetin, and 0.9994 for ECG.

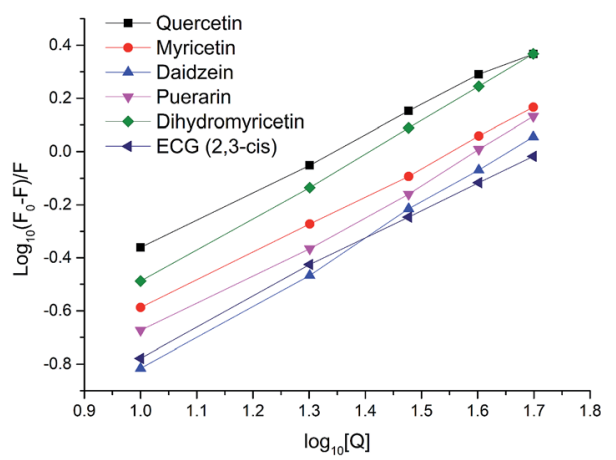

Fig. 3 Plots of $\log \left[\left(F_{0}-F\right) / F\right]$ versus $\log [Q]$ for quercetin, myricetin, daidzein, puerarin, dihydromyricetin, and ECG (2,3-cis). The degrees of fitting were 0.9985 for quercetin, 0.9991 for myricetin, 0.9987 for daidzein, 0.9964 for puerarin, 0.9996 for dihydromyricetin and 0.9988 for ECG.

flavonoids showed a wide range of $\log _{10} K_{\mathrm{a}}$ values (from 0.3808 to 14.019 ), and further comparison of $\log _{10} K_{\mathrm{a}}$ values would be described.

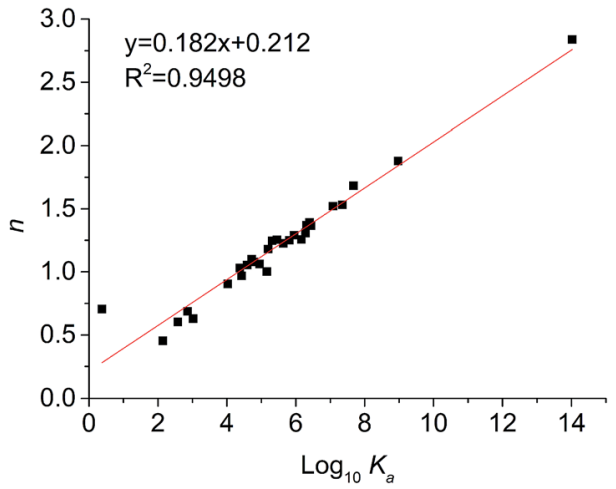

Fig. 4 Relationship between affinities $\left(\log _{10} K_{a}\right)$ and the number of binding sites $(n)$

\subsection{Structure-affinity relationship of flavonoids-xanthine oxidase interactions}

3.4.1. Methylation. Table 3 lists the effects of an additional methoxy group (hydrogen to methoxy group and hydroxyl group to methoxy group) in flavonoids on the affinities and inhibition for xanthine oxidase. Generally, the addition of a methoxy group in flavonoids weakened the binding affinities for xanthine oxidase by 268 to $10^{6}$ times. At the extreme, the affinity of genistein for xanthine oxidase was found to be $10^{6}$ times higher than that of biochanin A, which was the methylated form at the $4^{\prime}$-position. This kind of trend was reported in the interaction between flavonoids and bovine $\gamma$-globulin. ${ }^{9}$ The methylation increased the hydrophobicity of flavonoids, and the hydrophobic interaction plays an important role in binding to enzymes. ${ }^{32}$ However, the methylation of flavone, apigenin, and tangeretin increased the affinities for xanthine oxidase. For the inhibition on xanthine oxidase, the addition of a methoxy group in flavonoids mostly increased the inhibitory activities.

3.4.2. Hydroxylation. Table 4 showed the effect of hydroxylation of flavonoids on affinities for xanthine oxidase. It could be seen that the hydroxylation on rings $\mathrm{A}, \mathrm{B}$, and $\mathrm{C}$ of flavonoids affected the binding affinities for xanthine oxidase. Hydroxylation on the 7-position of flavone and flavanone weakened binding affinities, while hydroxylation on the 5-position of flavonoids mostly (3 out of 4) decreased binding affinities. However, hydroxylation on the 3-position (2 out of 3 ) and 3 'position ( 2 out of 2 ) of flavonoids mostly ( 3 out of 4 ) improved binding affinities. In terms of inhibition, hydroxylation on the 7-, 3'-, and 4'-positions of flavonoids enhanced inhibitory activities on xanthine oxidase, while hydroxylation on the 3position ( 2 out of 3 ) of flavonoids improved inhibitory activities.

3.4.3. Glycosylation. The effect of glycosylation of flavonoids on the affinities for xanthine oxidase was investigated (Table 5). Herein, the involved sugar moieties were rhamnose, glucose-rhamnose, and neohesperidose. The 3,7-glycosylation of kaempferol increased the affinity of kaempferol for xanthine oxidase by 311 times. 3-Glycosylation of quercetin increased the affinity by 10.2 times, 7-glycosylation of naringenin increased the affinity by 73.3 times, while the inhibition of kaempferol, quercetin, and naringenin decreased after glycosylation. 
Table 3 Effects of methylation of flavonoids on affinities

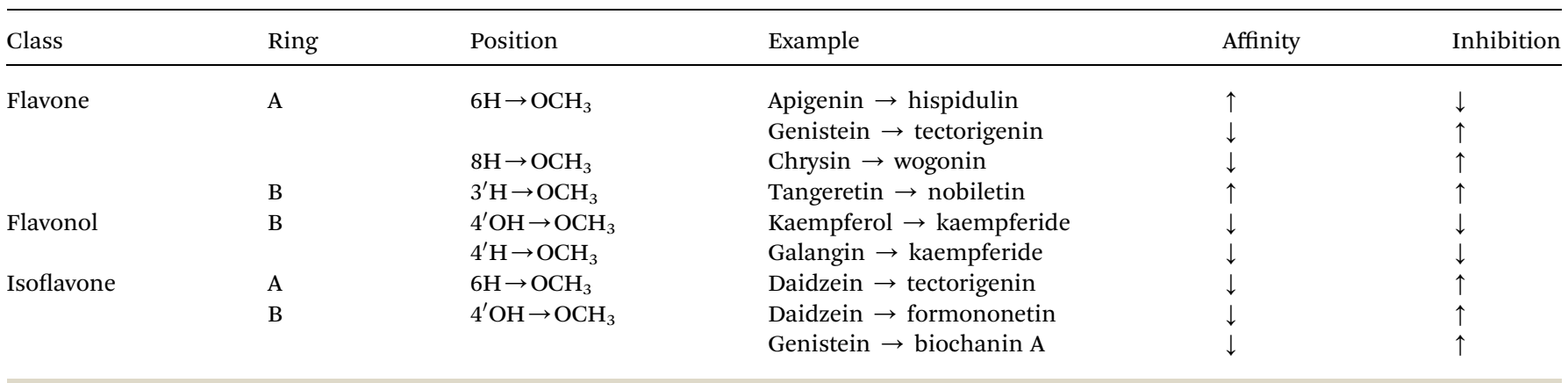

Table 4 Effects of hydroxylation of flavonoids on affinities

\begin{tabular}{|c|c|c|c|c|c|}
\hline Class & Ring & Position & Example & Affinity & Inhibition \\
\hline \multirow[t]{6}{*}{ Flavone } & A & $5 \mathrm{H} \rightarrow \mathrm{OH}$ & 7-OH flavone $\rightarrow$ chrysin & $\downarrow$ & $\uparrow$ \\
\hline & & $6 \mathrm{H} \rightarrow \mathrm{OH}$ & Chrysin $\rightarrow$ baicalein & $\uparrow$ & $\downarrow$ \\
\hline & & & Baicalin $\rightarrow$ baicalein & $\downarrow$ & $\uparrow$ \\
\hline & $\mathrm{B}$ & $3^{\prime} \mathrm{H} \rightarrow \mathrm{OH}$ & Apigenin $\rightarrow$ luteolin & $\uparrow$ & $\uparrow$ \\
\hline & & $4^{\prime} \mathrm{H} \rightarrow \mathrm{OH}$ & Chrysin $\rightarrow$ apigenin & $\downarrow$ & $\uparrow$ \\
\hline & & & Luteolin $\rightarrow$ quercetin & $\downarrow$ & $\uparrow$ \\
\hline \multirow[t]{4}{*}{ Flavonol } & A & $5 \mathrm{H} \rightarrow \mathrm{OH}$ & Fisetin $\rightarrow$ quercetin & $\downarrow$ & $\uparrow$ \\
\hline & $\mathrm{B}$ & $3^{\prime} \mathrm{H} \rightarrow \mathrm{OH}$ & Kaempferol $\rightarrow$ quercetin & $\uparrow$ & $\uparrow$ \\
\hline & & $4^{\prime} \mathrm{H} \rightarrow \mathrm{OH}$ & Galangin $\rightarrow$ kaempferol & $\uparrow$ & $\uparrow$ \\
\hline & & $5^{\prime} \mathrm{H} \rightarrow \mathrm{OH}$ & Quercetin $\rightarrow$ myricetin & $\downarrow$ & $\downarrow$ \\
\hline Isoflavone & A & $5 \mathrm{H} \rightarrow \mathrm{OH}$ & Daidzein $\rightarrow$ genistein & $\uparrow$ & $\downarrow$ \\
\hline
\end{tabular}

3.4.4. Hydrogenation of the $\mathrm{C}_{2}=\mathrm{C}_{3}$ double bond. The $\mathrm{C}_{2}=$ $\mathrm{C}_{3}$ double bond in conjugation with a 4-oxo group plays a very important role in the affinity for proteins. ${ }^{33}$ It was found in Table 5 that the hydrogenation of the $\mathrm{C}_{2}=\mathrm{C}_{3}$ double bond of flavonoids increased the binding affinities for xanthine oxidase. The affinities of dihydromyricetin and naringenin for xanthine oxidase were 7- and 4676-times higher than those of myricetin and dihydromyricetin apigenin, respectively. This trend is opposite to the affinities for $\alpha$-amylase and total plasma proteins. $^{17,33}$ The inhibition of dihydromyricetin and naringenin also decreased after hydrogenation of the $\mathrm{C}_{2}=\mathrm{C}_{3}$ double bond.

Table 5 Effects of glycosylation and hydrogenation of the $C_{2}=C_{3}$ double bond of flavonoids on affinities

\begin{tabular}{llll}
\hline & Example & Affinity & Inhibition \\
\hline Glycosylation & Kaempferol $\rightarrow$ kaempferitrin & $\uparrow$ & $\downarrow$ \\
& Quercetin $\rightarrow$ rutin & $\uparrow$ & $\downarrow$ \\
& Naringenin $\rightarrow$ naringin & $\uparrow$ & $\downarrow$ \\
Hydrogenation & Myricetin & $\uparrow$ & $\downarrow$ \\
of $\mathrm{C}_{2}=\mathrm{C}_{3}$ & $\rightarrow$ dihydromyricetin & & \\
& Apigenin $\rightarrow$ naringenin & $\uparrow$ & $\downarrow$
\end{tabular}

3.4.5. Catechins. Catechins are the major active components in green tea leaves, including (-)-C, (-)-GC, EC, (-)-ECG, (-)-EGC, (-)-EGCG, and (-)-GCG. The affinities between catechins and xanthine oxidase were determined, and the binding constants $\left(\log _{10} K_{\mathrm{a}}\right)$ of ECG, EGCG, and GCG for xanthine oxidase were found to be $4.7240,5.3153$, and 4.3766 , respectively (Table 2). However, C, EC, EGC, and GC were not able to quench the fluorescence of xanthine oxidase, and there was no binding constant value. This indicated the galloylated catechins have higher binding affinities for xanthine oxidase than nongalloylated catechins, and the presence of the galloyl moiety became the key factor. In addition, the affinity of gallocatechin gallate with a 2,3-trans structure was lower than that of gallocatechin gallate with a 2,3-cis structure. In terms of inhibition, the changes in inhibitory activities of catechins were not apparent.

\subsection{Comparison of trends in the binding affinities and inhibition}

Table 6 summarizes and compares trends in the binding affinities and inhibition of flavonoids during the structure modifications previously mentioned. In the methylation and hydroxylation of flavonoids in the A ring, the changes in 
Table 6 Comparison of trends in the binding affinities and inhibition of flavonoids for xanthine oxidase

\begin{tabular}{|c|c|c|c|c|c|c|c|c|}
\hline & & Samples & \multicolumn{2}{|c|}{ Affinity } & \multicolumn{2}{|c|}{ Inhibition } & \multicolumn{2}{|l|}{ Trends } \\
\hline & $\mathrm{B}$ & 5 & 1 & 4 & 1 & 4 & 3 & 2 \\
\hline \multirow[t]{2}{*}{ Hydroxylation } & A & 8 & 2 & 6 & 4 & 4 & 1 & 7 \\
\hline & B & 5 & 3 & 2 & 4 & 1 & 4 & 1 \\
\hline Hydrogenation of $\mathrm{C}_{2}=\mathrm{C}_{3}$ & & 2 & 2 & 0 & 0 & 2 & 0 & 2 \\
\hline
\end{tabular}

affinities and inhibition were almost all opposite (11 out of 12), while in the same structure modifications of flavonoids in the $\mathrm{B}$ ring, the changes of affinities and inhibition were almost all in accordance (7 out of 10). Moreover, in the glycosylation and hydrogenation of $\mathrm{C}_{2}=\mathrm{C}_{3}$ of flavonoids, the changes of affinities and inhibition were all opposite. Based on these findings, it might be concluded that the affinities for xanthine oxidase and the inhibition on xanthine oxidase would change in the opposite direction during methylation and hydroxylation of flavonoids in the $\mathrm{A}$ ring, and glycosylation and hydrogenation of $\mathrm{C}_{2}=\mathrm{C}_{3}$. However, the affinities and inhibition for xanthine oxidase would change in the same direction during methylation and hydroxylation of flavonoids in the $\mathrm{B}$ ring.

\subsection{Relationship of partition coefficients and the binding affinities}

The partition coefficient is a quantitative descriptor of lipophilicity and is important in related pharmacokinetic research. The partition coefficient values $\left(X \log P_{3}\right)$ of flavonoids could be obtained through the PubChem website (https://pubchem.ncbi.nlm.nih.gov/compound/). As shown in Fig. 5, the $X \log P_{3}$ values of flavonoids were inversely proportional to the corresponding $\log _{10} K_{\mathrm{a}}$ values. The linear regression curve was $X \log P_{3}=-0.073 \log _{10} K_{\mathrm{a}}+$

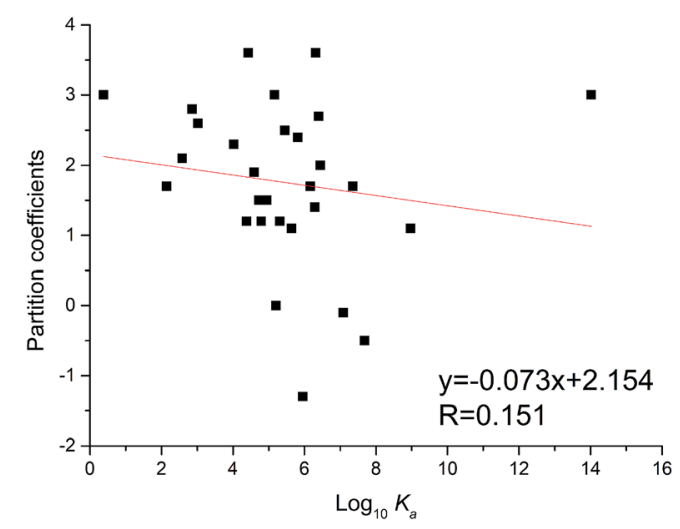

Fig. 5 Relationship between binding constants $\left(\log _{10} K_{\mathrm{a}}\right)$ and partition coefficients $\left(X \log P_{3}\right)$ of flavonoids.

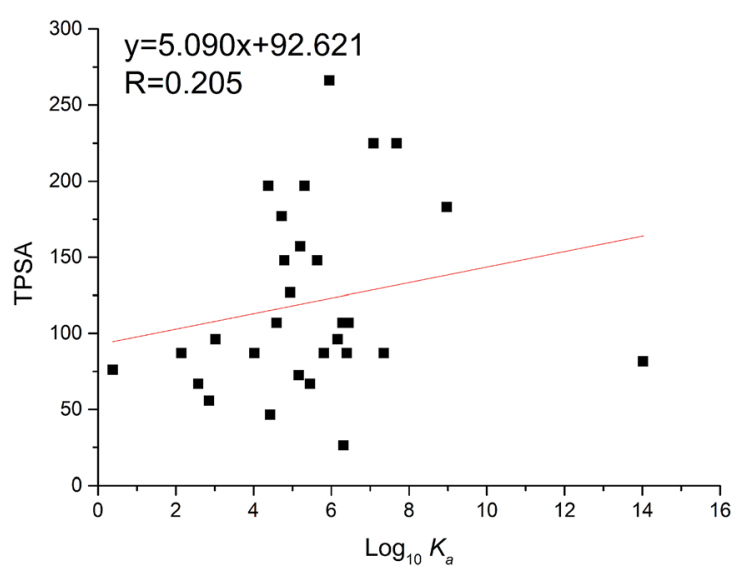

Fig. 6 Relationship between binding constants $\left(\log _{10} K_{\mathrm{a}}\right)$ and TPSA of flavonoids.

$2.154(R=0.151)$. The trend was the same as in the reported literature,$^{34}$ while the degree of fitting was a little lower.

\subsection{Relationship of topological polar surface area (TPSA) and binding affinities}

The TPSA was defined as the sum of surfaces of polar atoms in a molecule. This property has been shown to correlate with the human intestinal absorption, Caco-2 monolayers permeability and blood-brain barrier penetration. ${ }^{19}$ The relationship between TPSA and the binding affinity for proteins and enzymes were often studied. Herein, the TPSA values of flavonoids were also obtained through the PubChem website (https:// pubchem.ncbi.nlm.nih.gov/compound/). As a result, TPSA values of flavonoids were proportional to $\log _{10} K_{\mathrm{a}}$ values (Fig. 6). The linear regression curve was TPSA $=5.090 \log _{10} K_{\mathrm{a}}+92.621$ $(R=0.205)$, which is consistent with reported trend..$^{32}$ This might illustrate that flavonoids with high TPSA bind tightly with xanthine oxidase.

\section{Conclusions}

Based on the investigation of the interactions between 35 dietary flavonoids and xanthine oxidase, much analysis has been performed on the relationship between the structure properties of dietary flavonoids and their affinities for xanthine oxidase. 
Some structural influences on the affinities were concluded; methylation of hydroxyl groups weakened the affinities for xanthine oxidase and hydroxylation at the 7- and 5-positions weakened the binding affinities. However, hydroxylation at the 3- and 3'-positions mostly improved binding affinities. Glycosylation hydrogenation of the $\mathrm{C}_{2}=\mathrm{C}_{3}$ double bond also increased the affinities for xanthine oxidase. In addition, galloylated catechins showed higher binding affinities than nongalloylated catechins. The trends in the binding affinities and inhibition of flavonoids during the structure modifications were summarized; the affinities for xanthine oxidase and inhibition on xanthine oxidase change in the opposite direction during the methylation and hydroxylation of flavonoids in the A ring, and the glycosylation and hydrogenation of $\mathrm{C}_{2}=\mathrm{C}_{3}$. However, the affinities and inhibition for xanthine oxidase change in the same direction during the methylation and hydroxylation of flavonoids in the B ring. In order to further explore the effects of methylation in terms of the binding and inhibition of proteins, some flavonoids and their methylation derivatives were selected as the studied samples. The inhibition and binding parameters, using further spectroscopic methods, inhibition kinetics and docking analyses, of several enzymes were subject to investigation.

\section{Conflicts of interest}

There are no conflicts to declare.

\section{Acknowledgements}

This work was supported by the risk assessment of agricultural products quality and safety project (GJFP2019010) and the Natural Science Foundation of Hunan Province (2017JJ3348).

\section{References}

1 H. Cao, L. Chen and J. Xiao, Mol. Biol. Rep., 2010, 38, 22572262.

2 W. Jiang and M. Hu, RSC Adv., 2012, 2, 7948-7963.

3 J. Xiao, G. Kai, K. Yamamoto and X. Chen, Crit. Rev. Food Sci. Nutr., 2013, 53, 818-836.

4 F. Yang, Y. Zhao, G. Kai and J. Xiao, Food Funct., 2011, 2, 137141.

5 H. Rimac, Ž. Debeljak, D. Šakić, T. Weitner, M. Gabričević, V. Vrček, B. Zorc and M. Bojić, RSC Adv., 2016, 6, 7501475022.

6 J. Xiao, L. Chen, F. Yang, C. Liu and Y. Bai, J. Hazard. Mater., 2010, 182, 696-703.

7 J. Xiao, T. Chen, L. Chen, H. Cao, F. Yang and Y. Bai, J. Inorg. Biochem., 2010, 104, 1148-1155.

8 J. B. Xiao, Y. R. Zhao, H. Wang, Y. M. Yuan, F. Yang, C. Zhang and G. Y. Kai, Integr. Biol., 2011, 3, 1087-1094.
9 J. Xiao, G. Kai, F. Yang, C. Liu, X. Xu and K. Yamamoto, Mol. Nutr. Food Res., 2011, 55, S86-S92.

10 J. Xiao, H. Cao, Y. Wang, K. Yamamoto and X. Wei, Mol. Nutr. Food Res., 2010, 54, S253-S260.

11 B. Tu, Z.-F. Chen, Z.-J. Liu, R.-R. Li, Y. Ouyang and Y.-J. Hu, RSC Adv., 2015, 5, 73290-73300.

12 J. R. Lakowicz and B. R. Masters, Naturwissenschaften, 1991, 78, 456.

13 A. Papadopoulou, R. J. Green and R. A. Frazier, J. Agric. Food Chem., 2005, 53, 158-163.

14 J. Xiao, J. Shi, H. Cao, S. Wu, F. Ren and M. Xu, J. Pharm. Biomed. Anal., 2007, 45, 609-615.

15 H. Cao, L. Chen and J. Xiao, Mol. Biol. Rep., 2011, 38, 22572262.

16 J. Xiao, H. Cao, Y. Wang, K. Yamamoto and X. Wei, Mol. Nutr. Food Res., 2010, 54, S253-S260.

17 J. Xiao, Y. Zhao, H. Wang, Y. Yuan, F. Yang, C. Zhang and G. Kai, Integr. Biol., 2011, 3, 1087-1094.

18 J. Xiao, Y. Zhao, H. Wang, Y. Yuan, F. Yang, C. Zhang and K. Yamamoto, J. Agric. Food Chem., 2011, 59, 10747-10754.

19 F. Yang, Y. Zhao, G. Kai and J. Xiao, Food Funct., 2011, 2, 137141.

20 Y. Dong, H. Huang, M. Zhao, D. Sun-Waterhouse, L. Lin and C. Xiao, J. Funct. Foods, 2016, 24, 26-36.

21 A. Nagao, M. Seki and H. Kobayashi, Biosci., Biotechnol., Biochem., 1999, 63, 1787-1790.

22 T. Hatano, T. Yasuhara, R. Yoshihara, Y. Ikegami, M. Matsuda, K. Yazaki, I. Agata, S. Nishibe, T. Noro, M. Yoshizaki and T. Okuda, Planta Med., 1991, 57, 83-84.

23 J. Xiao, F. Wang, J. Liu, L. Wang, G. Kai and X. Yu, Mol. BioSyst., 2011, 7, 2452-2458.

24 F. Rasoulzadeh, H. N. Jabary, A. Naseri and M. R. Rashidi, Spectrochim. Acta, Part A, 2009, 72, 190-193.

25 L. L. Liu, A. P. Xiao, L. Ma and D. F. Li, J. Braz. Chem. Soc., 2017, 28, 360-366.

26 L. Liu, M. Yuan, S. Huang, J. Li, D. Li and L. Zhao, Appl. Sci., 2018, 8, 158.

27 X. Tang, P. Tang and L. Liu, Molecules, 2017, 22, 1036.

28 G. H. Wang, X. Y. Huang, D. Pei, W. D. Duan, K. J. Quan, X. T. Li and D. L. Di, New J. Chem., 2016, 40, 3885-3891.

29 B. Tu, Z.-J. Liu, Z.-F. Chen, Y. Ouyang and Y.-J. Hu, RSC Adv., 2015, 5, 106171-106181.

30 S. Li, L. Tang and H. Bi, Luminescence, 2016, 31, 442-452.

31 J. Xiao, E. Capanoglu, A. R. Jassbi and A. Miron, Crit. Rev. Food Sci. Nutr., 2016, 56, S29-S45.

32 J. Xiao, H. Cao, T. Chen, F. Yang, C. Liu and X. Xu, Biochimie, 2011, 93, 134-140.

33 J. Xiao, G. Kai, X. Ni, F. Yang and X. Chen, Mol. BioSyst., 2011, 7, 1883-1890.

34 J. Xiao, T. Chen, H. Cao, L. Chen and F. Yang, Mol. Nutr. Food Res., 2010, 55, 310-317. 